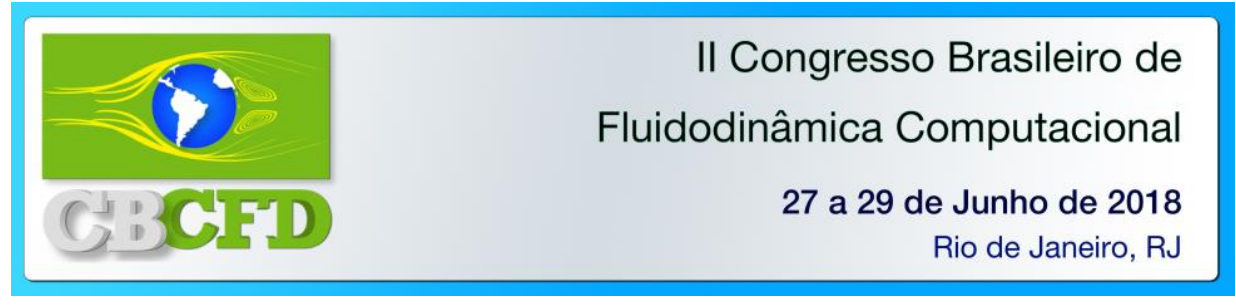

\title{
SIMULAÇÃO VIA CFD DA POLIMERIZAÇÃO DE PROPILENO EM UM REATOR TUBULAR EM LOOP
}

\author{
C. L. M. SILVA ${ }^{1}$, L. L. G. HASTENREITER ${ }^{1}$, M. R. C. TOMAZ ${ }^{1}$, W. M. POUBEL ${ }^{1}$ \\ ${ }^{1}$ Universidade Federal do Espírito Santo, Departamento de Engenharia Química \\ E-mail para contato: willian.poubel@ufes.br
}

\begin{abstract}
RESUMO - A ferramenta de Fluidodinâmica Computacional (CFD) foi utilizada para a análise do comportamento fluidodinâmico em um reator tubular do tipo loop, diante de uma reação de polimerização. Os resultados foram obtidos utilizando velocidades de entrada de 10 e $15 \mathrm{~m} / \mathrm{s}$ nas simulações, traçando-se perfis de velocidade e concentração. Em ambos casos, os perfis de velocidade gerados foram semelhantes; já os perfis de concentração se divergiram, havendo uma melhor conversão de polímero na velocidade de $10 \mathrm{~m} / \mathrm{s}$. Foi possível analisar uma melhor posição do tubo de saída através da análise gráfica de conversão.
\end{abstract}

\section{INTRODUÇÃO}

O uso de reatores tubulares para a produção de polímeros aparece como uma alternativa para as indústrias. Segundo Fogler (2009), as reações químicas nestes reatores ocorrem na medida em que os reagentes escoam pelo comprimento do tubo. Sua simplicidade geométrica garante menores custos fixo e operacional, além de possuírem elevado processamento de carga e elevada área de troca térmica (Nogueira, 2007; Rosa, 2013). Os reatores tubulares apresentam dificuldade no controle de temperatura de reações exotérmicas, ocasionando os chamados pontos quentes, podendo prejudicar a qualidade do produto final (Fogler, 2009).

Foram realizadas etapas de definição de geometria e malhas, identificação de fenômenos físico-químicos, especificação das condições de contorno e inicial, seleção das técnicas de solução numérica e discretização, resolução das equações algébricas através de métodos iterativos e análise da convergência e do resultado (Patel, 2007).

Foi analisado o comportamento da reação de polimerização de propileno de um reator loop em regime transiente. Foi usado o software ANSYS 17.0. O estudo em reatores loop via CFD possibilita a análise de detalhes específicos do processo (Lucca et al., 2007).

\section{MODELAGEM FLUIDODINÂMICA}

\subsection{Geometria}

A geometria do reator loop foi desenvolvida através do Design Modeler. A Figura 1 mostra o design. As dimensões adotadas para as "pernas" do reator foram de $70 \mathrm{~cm}$ de 


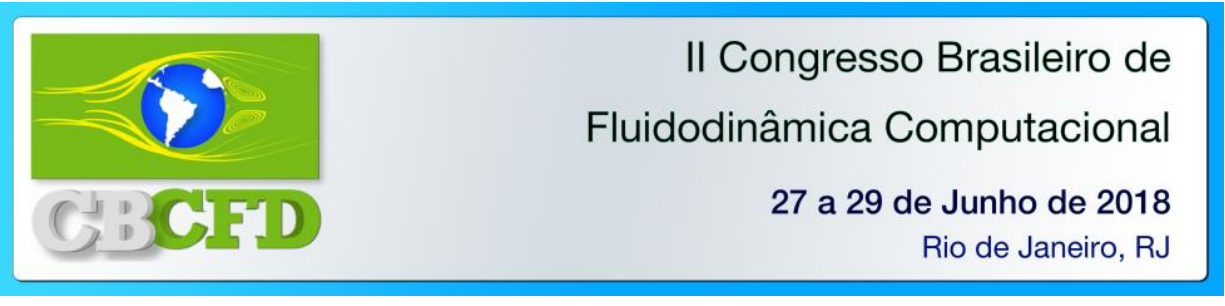

comprimento e conexões com $50 \mathrm{~cm}$ de comprimento. O diâmetro geral da tubulação (D) foi de $7,5 \mathrm{~cm}$ e o diâmetro dos tubos de entrada e saída (d) foi de $2,5 \mathrm{~cm}$.

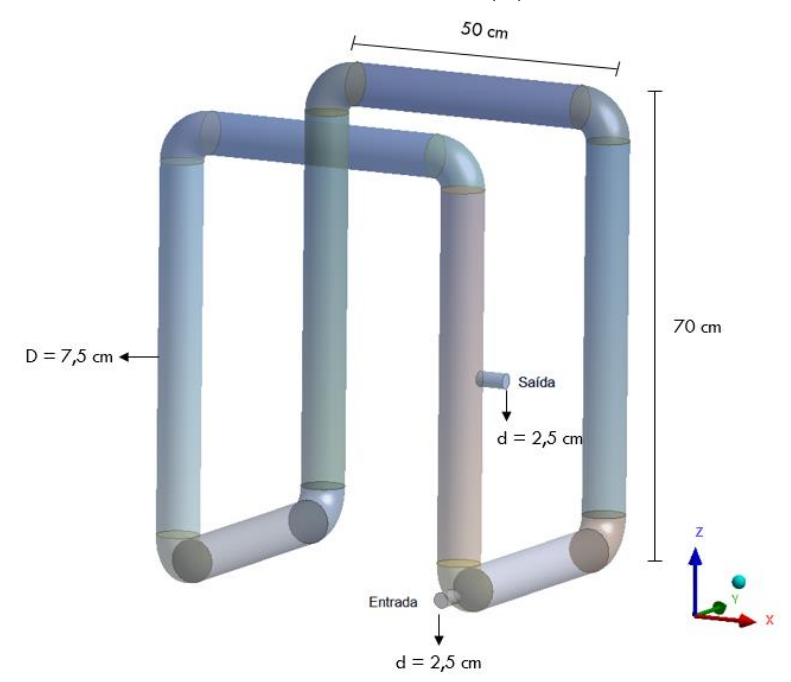

Figura 1 - Geometria adotada para o reator loop

\subsection{Definição da malha}

Foi gerada a malha no módulo Meshing, utilizando elementos tetraédricos. A função inflation foi utilizada a fim de se obter uma adaptação das regiões de extremidade da tubulação. Na Figura 2 é possível visualizar os elementos de malha na geometria.

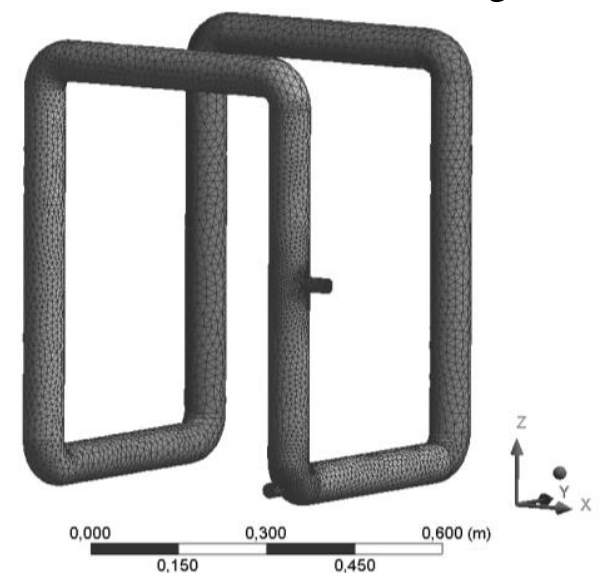

Figura 2 - Elementos de malha na geometria no reator loop

\subsection{Simulação}

As simulações foram realizadas no Fluent, foi adotado regime transiente, gravidade de $9,81 \mathrm{~m} / \mathrm{s}^{2}$ no eixo z, modelo k-ômega SST de turbulência, modelo de transporte volumétrico de espécies; e foram adotados inlet velocity $(10$ e $15 \mathrm{~m} / \mathrm{s})$ na entrada e pressure outlet (35 atm) na saída. O banco de dados de reações e compostos do módulo Fluent não possui polímeros e nem reações de polimerização. Desta forma, o composto polipropileno foi adicionado manualmente e a reação utilizada, inicialmente, foi simplificada seguindo a taxa reacional de Arrhenius. 


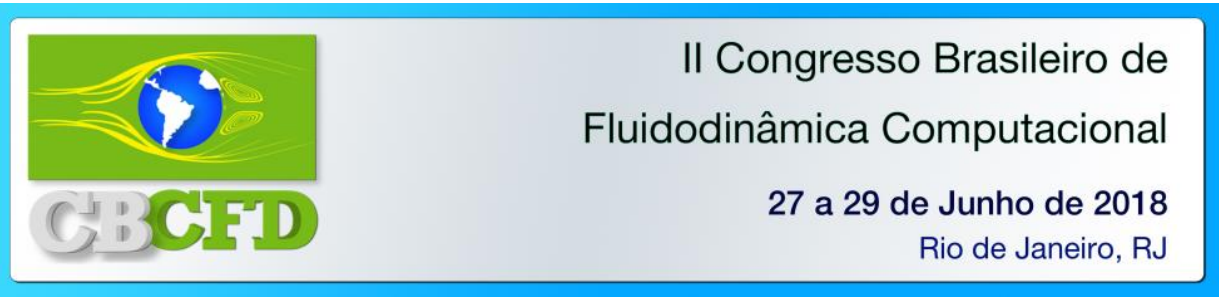

A cinética de polimerização mais condizente com a realidade foi implementada através de um código vinculado ao programa através da função User Defined Function (UDF). Os resultados apresentados neste trabalho descrevem dados obtidos a partir desta UDF, cuja escrita foi baseada em códigos disponibilizados por UDF Manual (2006). Foi considerado que o reator estava inicialmente cheio de propileno, operando a uma temperatura constante de $343,15 \mathrm{~K} \mathrm{e}$ pressão de 35 atm, estes baseados em estudos de Fontoura (2016). Os dados de velocidade de entrada foram baseados nos estudos de Machado, Cardozo e Jesus (2009). Foram adotadas velocidades de entrada de $10 \mathrm{~m} / \mathrm{s}$ e $15 \mathrm{~m} / \mathrm{s}$.

\section{RESULTADOS E DISCUSSÃO}

Foram realizadas duas simulações com velocidade de entrada de 10 e $15 \mathrm{~m} / \mathrm{s}$. Foi analisado os perfis de velocidade e concentração ao longo do reator, ao final do tempo reacional. Foram traçadas 8 linhas ao longo do reator e cada linha fornece dados de escoamento, 4 destas linhas foram selecionadas para análise.

As curvas de conversão nas quatro linhas são mostradas abaixo na Figura 3.

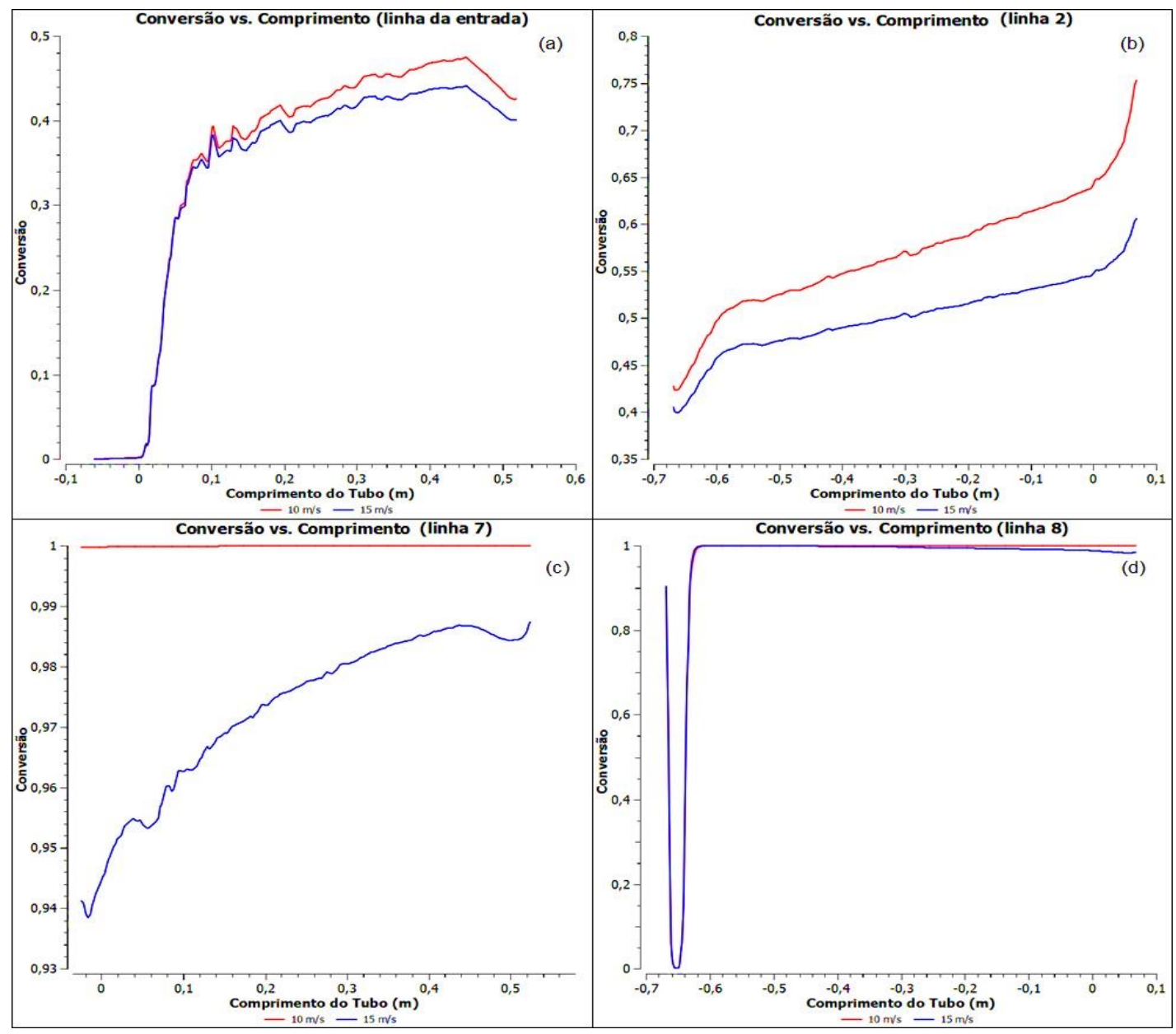

Figura 3 - Curvas de perfis de conversão nas linhas (a) 1, (b) 2, (c) 7 e (d) 8 .

É possível observar que as diferenças de conversão começam sutis na Figura 5a, representante da linha 1 , depois se diferenciam mais a partir da Figura 3b, onde a fração para 


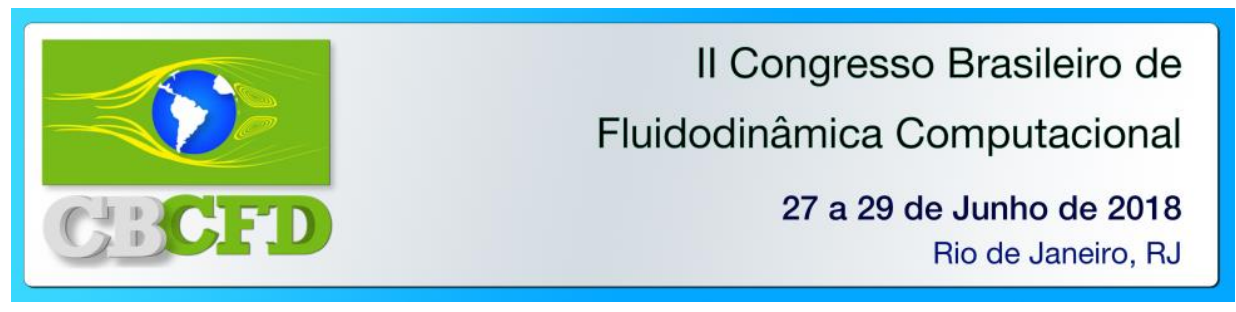

$10 \mathrm{~m} / \mathrm{s}$ é maior que para $15 \mathrm{~m} / \mathrm{s}$. Na Figura $3 \mathrm{c}$ observa-se que já foi alcançada conversão total para velocidade de $10 \mathrm{~m} / \mathrm{s}$. Por fim, as frações se aproximam na Figura 3d. Fica claro que a conversão para a velocidade de $10 \mathrm{~m} / \mathrm{s}$ foi mais elevada e a igualdade na última curva se deve à conversão máxima alcançada pelos dois perfis na linha 8 .

\section{CONCLUSÕES}

Foi possível prever a conversão de uma reação de polimerização em um reator tubular loop. Foi possível testar a geometria inspirada no reator de Fontoura (2016) e a malha gerada. Os resultados obtidos permitiram a identificação de importantes zonas de baixas velocidades e de diferentes concentrações no reator para diferentes velocidades de entrada.

Para melhora do escoamento ao longo do tubo sugere-se a inserção de anteparos dentro do reator nas zonas de menor velocidade do escoamento. Através da análise comparativa podese observar que a melhor velocidade de entrada é a de $10 \mathrm{~m} / \mathrm{s}$ e que a tubulação da linha 7 (última seção do reator) é um possível lugar para se posicionar a saída, já que a conversão já é máxima nessa região.

\section{REFERÊNCIAS BIBLIOGRÁFICAS}

FOGLER, H. S. Elementos de engenharia das reações químicas. LTC, 2009.

FONTOURA, T. B. Modelagem de um reator tubular bifásico do tipo LOOP para a polimerização do propeno. Tese (Doutorado em Engenharia Química). Universidade Federal do Rio de Janeiro, Rio de Janeiro, 2016.

LUCCA, E. A. et al. Modelagem e simulação de reatores industriais em fase liquida do tipo Loop para polimerização de propileno. 2007. Tese de Doutorado.

MACHADO, A. R.; CARDOZO, N. S. M.; JESUS, M. A. S. Modelagem do escoamento em um reator loop. In CONGRESSO BRASILEIRO DE POLÍMEROS, 10, 2009, Foz do Iguaçu. Anais.

NOGUEIRA, A. L. Desenvolvimento de reator tubular para a produção de polímeros em suspensão. 2007. Tese de Doutorado. UNIVERSIDADE FEDERAL DE SANTA CATARINA.

PATEL, H. Computational fluid dynamics (CFD) analysis of mixing in styrene polymerization. 147 f. Dissertação (Mestrado em Engenharia Química) - Universidade de Ryerson, Toronto, 2007.

ROSA, I. da S. Análise dinâmica e de estabilidade de reatores tubulares de polimerização de propeno do tipo loop. 2013. Tese (Doutorado em Engenharia Química). Universidade Federal do Rio de Janeiro, Rio de Janeiro, 2013.

UDF MANUAL. Fluent 6.3. 2006. ANSYS, Inc. 